\title{
Guillain-Barre Syndrome with Covid-19 Infection
}

\author{
Savita Bansiram Pohekar ${ }^{1}$, Amruta Shalikram Kothe ${ }^{2}$ \\ 1,2 Department of Medical Surgical Nursing, Datta Meghe Institute of Medical \\ Sciences(Deemed to Be University) Smt. Radhikabai Meghe Memorial College of \\ Nursing, Sawangi (Meghe), Wardha, Maharashtra, India.
}

\section{INTRODUCTION}

COVID-19 from Wuhan, China has spread easily all over the world. Most of the COVID-19 infected patients have fever and breathing disorders. Here, we report a case of Guillain-Barre syndrome (GBS) with a COVID-19 infection. GBS is a very rare disease with corona virus infection. It is really hard to diagnose. In this state the limbs of the patient are slowly weakened. The condition worsens daily with weakness of the limbs. The Guillain-Barre syndrome is a complex and acute or chronic neurological condition. Campylobacter jejuni and other viruses, including cytomegalovirus and Epstein Barr Virus, are causing this condition. ${ }^{1}$

It is a disorder that is progressive, symmetric, proximate, distal, and characterised by weakness. Muscle reflexes are reduced to absent. Aetiology is unclear, Death is uncommon. The diagnosis of GBS can be made by cerebrospinal fluid analysis and nerve conduction studies. ${ }^{2}$

We present a case of Guillain-Barre syndrome with COVID-19 infection, who presented to the emergency Outpatient department with complaints of weakness against his bilateral upper and lower limb.

\section{PRESENTATION OF CASE}

A 16-year-old male patient was admitted to our hospital in medicine ICU with chief complaints of weakness in his bilateral upper and lower limbs, trouble with swallowing, cough, and widespread fatigue. There was no recent history of travel. He has been reporting shortness of breath and cough for one week. He took tablet crocin and cough syrup to relieve his symptoms. When presented first in the emergency room, the chief complaints were weakness over the bilateral lower limb, difficulty in deglutition, difficulty in coughing, and generalized weakness. On admission, he was febrile (100 - degree centigrade), with moderate tachycardia, oxygen saturation was $98 \%$, and haemodynamically stable. On physical examination, he had a stiff neck, the ranges of movement were active, the spinal cord was compromised, and it had damaged the vagus nerve. It disrupted weakness and control of muscle strength. Counting of the white cells, sodium, and potassium, renal and liver functions were usual. He was tested positive with SARS - CoV - 2 in RT - PCR by the oropharyngeal swab. The infection isolation room of the ICU was immediately provided and started with supportive treatment and medications. Tablet Arbidol, tablet lopinavir, and tablet ritonavir.
Corresponding Author: Savita Bansiram Pohekar, Medical Surgical Nursing, Radhikabai Meghe Memorial College of Nursing, Sawangi (Meghe), Wardha, Maharashtra, India.

E-mail: savitaak15@gmail.com

DOI: $10.14260 / \mathrm{jemds} / 2021 / 437$

How to Cite This Article:

Pohekar SB, Kothe AS. Guillain Barre syndrome with Covid-19 Infection. J Evolution Med Dent Sci 2021;10(28): 2139-2141 DOI:

$10.14260 /$ jemds/2021/437

Submission 07-02-2021,

Peer Review 12-05-2021,

Acceptance 19-05-2021,

Published 12-07-2021.

Copyright (C) 2021 Savita Bansiram Pohekar et al. This is an open access article distributed under Creative Commons Attribution License [Attribution 4.0 International (CC BY 4.0)] 
Meningism, sensory level of the spinal cord, bladder or bowel involvement, or dysautonomia were not present. His brain spinal fluid (CSF) showed cytoalbuminological Protein $>1.25$ g / L. GBS has been confirmed after Magnetic Resonance Imaging (MRI) and CSF examination. In the first week of admission in the intensive care unit, the patient was monitored, and he required a nasogastric tube for food because he had trouble in swallowing, without a breathing disorder. He got Intravenous immunoglobulin (IVIG) $0.4 \mathrm{~g} /$ $\mathrm{kg}$ per day for five days and his symptoms stabilized. $\mathrm{He}$ received antibiotics Tablet - Augmentin 625mg, tabletcetirizine $10 \mathrm{mg}$, tablet Pantocid $40 \mathrm{mg}$, tablet Mucomix 600mg, Nasal drop - Xylomist, Nebulization with Dublin and Budecort 8 hourly. He was stable at the time of discharge with no further complications.

\section{NURSING DIAGNOSIS}

- Ineffective breathing pattern related to ascending weakness as evidenced by respiratory changes.

- Acute pain related to inflammation of nerves as evidenced by pain scale assessment.

- Impaired physical mobility related to neuromuscular impairment, as evidenced by decreased muscle strength.

- Anxiety-related to change in health status and a threat to self-concept as evidenced by expression of helplessness.

- Disturbed sleep pattern related to hospitalization as evidenced by patient verbalization.

\section{DISCUSSION OF MANAGEMENT}

The nurse is responsible for prescribing medicines and evaluating the positive and negative effects. The combination of these effects determines the type of pharmacological therapy and the dosage. Clinical efficacy assessment measures for nursing includes, to note breathing fatigue symptoms, including cough and shortness of breath, perform a movement exercise aggressive, passive, and isotonic, maintain records of intakes and outputs. The priority diagnosis is based on the condition of the patient.

Our patient had classical GBS symptoms that had started approximately two weeks after respiratory tract infection with COVID-19. This disorder was confirmed by electro diagnostic findings. Corona virus studies have shown that these viruses are neurotrophic and neuroinvasive.

Kim. JE et al. in their study reported neurological symptoms of COVID - 19 infection. Other beta-coronaviruses (SARS and MERS), including polyneuropathy, myopathy, stroke and GBS, also reported neurological events. ${ }^{3}$

Mayanja M. Kajumba et al. in their study showed that COVID - 19, SARS - CoV-2 was a possible GBS cause that occured not only in those serious but also in patients who had moderate symptoms or had no symptoms of COVID-19. Although GBS is generally considered a post-infectious neurological condition, many COVID-19 patients with an atypical para-infectious profile have a brief delay between the onset of COVID-19 and GBS in most of the cases, including post-infectious patients. ${ }^{4}$
In the present case, the problem arised from a coincidence or probability that the COVID - 19 infection was associated with the Guillain-Barré syndrome. Before the COVID-19 symptoms began on day 4 of weakness, our patient developed Guillain-Barré syndrome signs. The question arises whether this could be a para-infectious phenomenon because of the virus' direct neuroinvasion. Or will the time of incubation be post-infectious for respiratory symptoms that overlap for neurological symptoms that mediate immune? Guillain-Barré Syndrome usually follows a day 1 to 4 week's late immune stimulus.

Our patient responded heavily to immunoglobulin therapy, emphasizing the importance of early detection and treatment. 5

GBS has significant implications, and early identification is essential to track ambulatory failure and immunoglobulin therapy initiation. We should not overlook the viral myositis in patients with paraesthesia and mobilization problems after the COVID-19 signs. We should treat GBS as potentially rare with severe complications for weeks after the initial COVID19 infection. $^{6}$

Further research for GBS mechanism in patients with covid-19 should be carried out in the future.

Overall, this study only reported an association of Guillain-Barré and SARS-CoV-2. Infection, and further, it needs cases of epidemiological knowledge to promote a fundamental association.

\section{Outcome and Follow-Up}

In the first week of IVIG administration, the motor symptoms of the patient began to improve. His motor strength had improved four weeks later. Exchange of plasmas and immunoglobulin therapies were effective. The patient was improving with the help of nursing care and physiotherapy, it increased strength of muscles by enhancing and mobilizing. Improve balance and mobility by evaluating various mobility aids and postural retraining. Reduce muscle rigidity, spasms, and pain by stretching programs.

\section{CONCLUSIONS}

This is a rare clinical incidence of GBS initially triggered by sensory disruption (loss of cold and hot sensation). While this is not the first such case, in the literature, we assume the study may lead to improved understanding. Early detection is important to track for ambulation failure and initiation of immunoglobulin therapy. We should consider GBS a possible unusual but severe complication. Paresthetic and mobilization difficulties should not be ignored as viralassociated myositis in patients after COVID-19 symptoms.

Financial or other competing interests: None.

Disclosure forms provided by the authors are available with the full text of this article at jemds.com.

\section{REFERENCES}

[1] Mazidi M, Imani B, Norouzy A, et al. Guillain barre syndrome: a case report. Int J Hosp Res 2013;2(2):91-3. 
[2] Sahin AR, Erdogan A, MutluAgaoglu P, et al. 2019 Novel coronavirus (COVID-19) outbreak: a review of the current literature. EJMO 2020;4(1):1-7.14.

[3] Kim JE, Heo JH, Kim HO, et al. Neurological complications during treatment of Middle-East respiratory syndrome. J Clin Neurol 2017;13(3):227-33.

Kajumba MM, Kolls BJ, Koltai DC, et al. COVID-19associated guillain-barre syndrome: atypical parainfectious profile, symptom overlap and increased risk of severe neurological complications. SN Compr Clin Med 2020:1-13.

[4] Assini A, Benedetti L, Di Maio M, et al. New clinical manifestation of COVID-19 related Guillain-Barrè syndrome highly responsive to intravenous immunoglobulins: two Italian cases. Neurol Sci 2020;41(7):1657-8.

[5] Coronavirus statement. The Encephalitis Society. [Cited 2021 Feb

18]. 